\title{
PDGF-D in renal fibrosis
}

Kidney fibrosis is a hallmark and common end point of numerous chronic kidney diseases, but effective therapies to treat kidney fibrosis are lacking. Now, Peter Boor and co-workers show that an isoform of platelet-derived growth factor, PDGF-D, is dispensable for renal development and function but mediates renal fibrosis, and might represent an attractive therapeutic target. "We had previously shown that PDGF-D is a crucial factor involved in mesangioproliferative glomerulonephritis, but its role in renal fibrosis was unknown," explains Boor.

In their recent study the researchers found that in human and murine fibrotic kidneys, PDGF-D and its receptor PDGFR- $\beta$ are overexpressed in activated mesenchymal cells, and PDGF-D is expressed de novo in tubular cells. In addition, they showed that the levels of PDGF-D drive kidney fibrosis; PDGF-D overexpression in healthy mice increased the deposition of interstitial collagen, whereas genetic depletion of PDGF-D significantly reduced fibrosis in two murine models of renal scarring. According to Boor, PDGF-D drives fibrosis by specifically affecting renal PDGFR- $\beta^{+}$mesenchymal cells but not inflammation.

"As PDGF-D was dispensable for normal kidney development and physiological functions" says Boor, "PDGF-D inhibition could be particularly suitable for a dual-hit therapy for progressive mesangioproliferative diseases, where PDGF-D drives both the glomerular mesangioproliferative changes and interstitial fibrosis, such as in IgA nephropathy."

Boor cautions that the role of the second PDGFR- $\beta$ ligand, PDGF- $B$, and its complementarity or dissimilarities to PDGF-D remain elusive. "The potential differential roles of these two ligands and their potential interplay should be clarified before clinical trials are initiated."

Andrea Aguilar

ORIGINAL ARTICLE Buhl, E. M. et al. The role of PDGF-D in healthy and fibrotic kidneys. Kidney Int. http://dx.doi.org/ 10.1016/j.kint.2015.12.037

FURTHER READING Kok, H. M., et al. Targeting CTGF, EGF and PDGF pathways to prevent progression of kidney disease. Nat. Rev. Nephrol. 10, 700-711 (2014) 